\title{
Effect of Resveratrol on periodontal pathogens during experimental periodontitis in rats
}

\section{Fabiano Ribeiro CIRANO(a) Renato Correa Viana CASARIN(a) Fernanda Vieira RIBEIRO(a) Marcio Zaffalon CASATI(a) Suzana Peres PIMENTEL (a) Tiago TAIETE(b) \\ Maria Martha BERNARDI ${ }^{(a)}$}

(a) Universidade Paulista, School of Dentistry, Dental Research Division, São Paulo, SP, Brazil.

(b) Universidade Estadual de Campinas - UNICAMP, School of Dentistry at Piracicaba, Department of Prosthodontics and Periodontics, Piracicaba, SP, Brazil.
Declaration of Interests: The authors certify that they have no commercial or associative interest that represents a conflict of interest in connection with the manuscript.

\section{Corresponding Author:}

Fabiano R. cirano

E-mail: cirano.fabiano@gmail.com

DOI: 10.1590/1807-3107BOR-2016.vol30.0128

Submitted: Nov 13, 2015

Accepted for publication: Aug 16, 2016

Last revision: Sep 22, 2016

\begin{abstract}
The objective of this study was to investigate the antibacterial effect of resveratrol against putative periodontal pathogens during the progression of experimental periodontitis in rats. Periodontitis was induced in rats in one of the first molars chosen to receive a ligature. Animals were assigned to one of two groups: daily administration of the placebo solution (control group, $\mathrm{n}=12$ ) or $10 \mathrm{mg} / \mathrm{Kg}$ of resveratrol (RESV group, $\mathrm{n}=12$ ). The therapies were administered systemically for 30 days, for 19 days before periodontitis induction and then for another 11 days. Then, the presence and concentrations of Porphyromonas gingivalis, Tannerella forsythia and Aggregatibacter actinomycetemcomitans in the cotton ligatures collected from the first molars were evaluated using real-time PCR. Inter-group comparisons of the microbiological outcomes revealed that no differences were detected for $P$. gingivalis, T. forsythia and A. actinomycetemcomitans levels ( $\mathrm{p}>0.05)$. Continuous use of resveratrol did not promote additional benefits in microbiological outcomes during experimental periodontitis in rats.
\end{abstract}

Keywords: resveratrol; periodontitis; alveolar bone loss; anti-inflammatory agents; anti-bacterial agents.

\section{Introduction}

Periodontitis is a major chronic inflammatory disease that is the most prevalent form of bone pathology in humans with up to $15 \%$ of the adult population affected by severe periodontitis at some point in their lives. ${ }^{1}$ This disease is characterized by an infection that leads to the occurrence of the destruction of the supporting tissue and induces a local production of immune-inflammatory mediators in response to periodontal pathogens and their products. ${ }^{2}$ Although the nature of host immune-inflammatory responses to microbiota may motivate the pathology that is observed, the existence of one or more microorganisms that are considered to be a periodontal pathogen in sufficient numbers is needed to establish periodontitis. ${ }^{3}$ Key periodontopathic bacteria, such as Porphyromonas gingivalis (Pg), Tannerella forsythia (Tf) and Aggregatibacter actinomycetemcomitans (Aa), are related to the beginning of periodontal disease and may prolong imbalance in the microbiota and the host response in periodontal tissues, which encourages the development of periodontitis. ${ }^{2,4,5}$ Thus, although there is a consensus that the response of the host against bacterial challenge is essential 
for the development of the disease, the specific etiological agent of periodontal disease is bacterial biofilm, which has been confirmed by experimental and human studies. ${ }^{4,6}$

The compound resveratrol (3,5,4'-trihydroxystilbene) is a polyphenol present in many plant species that are used for human consumption, particularly grapes and red wine. ${ }^{7}$ It has been shown by the scientific community that resveratrol has potent antioxidant, antitumor and anti-inflammatory effects that benefit the treatment of some diseases, such as cancer, diabetes, cardiovascular disorders, degenerative diseases, autoimmune diseases and metabolic diseases. $8,9,10,11,12$

The impact of the use of resveratrol on the immune-inflammatory system is closely linked to its antioxidant capacity and anti-inflammatory activity. ${ }^{11,13,14,15,16}$ However, some studies have also indicated that resveratrol has significant antimicrobial effects in distinct infectious circumstances. . $178,19,20,21$ As related to periodontitis, a study by $\mathrm{O}^{\prime}$ Connor et al. ${ }^{18}$ demonstrated the effect of resveratrol in vitro on the inhibition of periodontal A. actinomycetemcomitans and $P$. gingivalis. Park et al. ${ }^{17}$ also showed that resveratrol attenuated $P$. gingivalis LPS-induced monocyte adhesion to the endothelium, which suggested it has a therapeutic role in periodontal pathogen-induced vascular inflammation.

Due to the potential benefits of resveratrol for controlling periodontal disease $\mathrm{e}^{15,22}$ and taking into account its antimicrobial ability in different types of infectious diseases, ${ }^{19,20,21}$ the aim of this investigation was to determine the antibacterial effects of resveratrol against putative periodontal pathogens in a rat experimental periodontitis model.

\section{Methodology}

\section{Animals}

This study used male Wistar rats that were 10 weeks old and that weighed $308 \pm 35 \mathrm{~g}$ at the beginning of the study. The rats were obtained from the Butantan Institute in São Paulo, Brazil. The rats were acclimatized for 15 days before they were used, and they were kept in temperature-controlled cages that were exposed to a 24-h light-dark cycle with equal light and dark time; the animals had free access to water and food ad libitum and were housed at the Bioterium of Paulista University (Labina, Purina1, Paulinia, SP, Brazil). The experimental procedure was approved by the Paulista University Institutional Animal Care and Use Committee (060/11 CEP/ICS/UNIP).

\section{Study design and treatment groups}

The study design and treatment groups were previously described in detail. ${ }^{16}$ Briefly, animals were assigned to one of two groups: a control group $(\mathrm{n}=12)$ that received daily administration of a placebo solution for 30 days, or the RESV Group $(n=12)$ that received daily administration of $10 \mathrm{mg} / \mathrm{kg}$ of resveratrol for 30 days. A stock solution of resveratrol (R5010-500MG - Sigma-Aldrich Ltda, São Paulo, Brazil) (molecular weight: 228.2)) was prepared in ethanol and further diluted in water to working concentrations. The placebo solution was composed of the same quantities of ethanol and water that were used in preparing the resveratrol. The therapies were administered via gavage by a blinded researcher for 30 days - for 19 days before periodontitis induction and for another 11 days following ligature placement. Figure illustrates the study design.

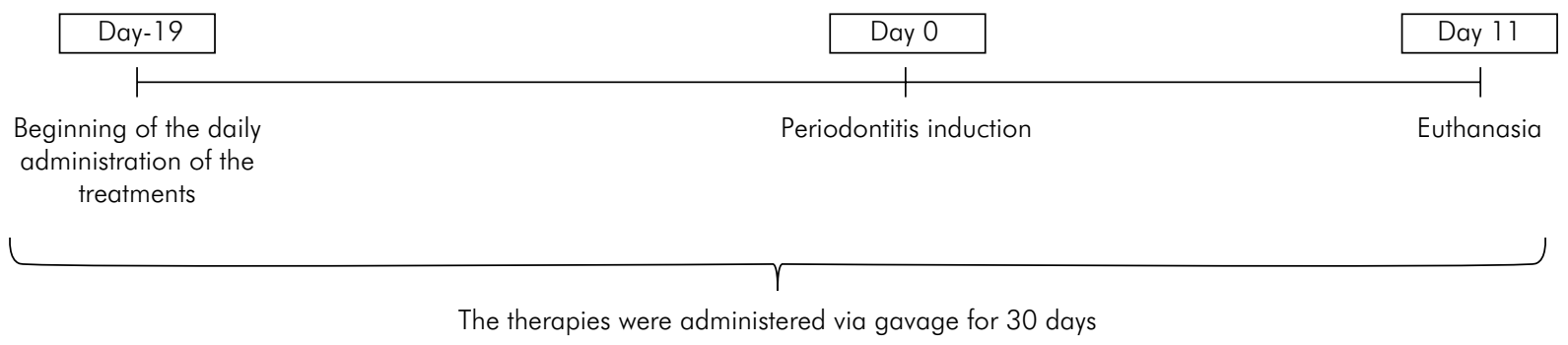

Figure. Schematic illustration of the study design. 


\section{Rat periodontitis model}

To induce experimental periodontitis, one of the mandibular first molars of each animal was randomly assigned to receive a cotton ligature (Corrente Algodão no. 10; Coats Corrente, São Paulo, Brazil) that was knotted submarginally in a cervical position. The ligatures were kept in position to allow biofilm accumulation over 11 days. ${ }^{23}$ The contralateral tooth was left unligated so that it could be used as a control for alveolar bone loss. This procedure was performed under general anesthesia by intramuscular administration of $0.5 \mathrm{~mL} / \mathrm{kg}$ ketamine hydrochloride (Dopalen ${ }^{\circledast}$, Agribrands Brasil Ltda., Paulínia, Brazil) and $10 \mathrm{mg} / \mathrm{kg}$ xylazine hydrochloride (Rompun ${ }^{\circledast}$, Bayer S.A., São Paulo, Brazil).

The animals were evaluated daily throughout the experiment to check for possible clinical or toxicological symptoms. Thirty days after the start of the study, the animals were euthanized by $\mathrm{CO} 2$ inhalation. The cotton ligatures were collected from the 1st molar from each rat for microbiological analysis.

The morphometric analysis of bone loss and immuno-enzymatic assay of the gingival tissue have been previously described in detail. ${ }^{15}$

\section{Microbiological evaluation}

Cotton ligatures were collected from the 1st molar of each rat and then were placed into sterile tubes containing $300 \mu \mathrm{L}$ of $0.5 \mathrm{mM}$ Tris-EDTA. DNA was extracted from each sample using a commercially available kit (QIAamp Genomic DNA Extraction, Qiagen).

The presence percentage of positive sites and the concentration of $P$. gingivalis, T. forsythia and A. actinomycetemcomitans were evaluated by real-time PCR using specific primers reported in the literature by a previous researcher. ${ }^{24,25}$ Initially, DNA was extracted from the subgingival biofilm. Real-time PCR was performed using the FastStart DNA Master SYBR Green I kit. The concentration of the DNA used in each run was $10 \mu \mathrm{g} /$ $\mathrm{ml}$. The amplification profiles were as follows: $95^{\circ} / 10$, $55^{\circ} / 5{ }^{\prime}, 72^{\circ} / 4 ;, 40$ cycles for $\mathrm{Pg} ; 95^{\circ} / 10^{\prime}, 55^{\circ} / 5{ }^{\prime}, 72^{\circ} / 3^{\prime}$ and 40 cycles for Aa. Absolute quantification of target bacteria in clinical samples was performed using Pg (ATCC 33277) and Aa (JP2) as controls. The determination of DNA genome copies in the controls was based on the genome size of each bacteria and the mean weight of one nucleotide pair with a minimum detection level of $10^{3}$ cells. The examiners that performed the microbiological evaluations were blinded to the experimental groups.

\section{Statistical analysis}

To test the null hypothesis that resveratrol had no influence on periodontal pathogens levels, intergroup analysis of the concentration of each bacteria was performed by unpaired Student's t-test, and comparisons between the groups of the percentage of sites that were positive for periodontal pathogens were performed using the Chi-square test. The significance level established for all analyses was $5 \%$.

\section{Results}

For the microbiological outcomes observed in this study, the inter-group comparisons revealed that there was no difference between groups for Aa, Pg or Tf levels ( $p>0.05)$. Similar results were observed regarding with the percentage of sites that were positive for these bacteria after therapy, and there were no differences between groups $(p>0.05)$. Tables 1 and 2 show the concentration $(\log [])$ and the frequency (\%), respectively, of each of the periodontal pathogens that were tested.

Table 1. Mean \pm standard deviation of the concentration (log [ ]) of the periodontal pathogens Aggregatibacter actinomycetemcomitans $\mathrm{Aa}$, Porphyromonas gingivalis $\mathrm{Pg}$ and Tannerella forsythia Tf in ligatures from the control and RESV groups.

\begin{tabular}{lccc}
\hline Variable & $\mathrm{Aa}$ & $\mathrm{Pg}$ & Tf \\
\hline Control $[\%(\mathrm{n})]$ & $6.6 \pm 1.0$ & $2.4 \pm 2.4$ & $2.1 \pm 2.4$ \\
RESV [\% (n)] & $5.4 \pm 1.8$ & $1.5 \pm 2.2$ & $1.5 \pm 2.1$ \\
p-value & 0.08 & 0.39 & 0.53 \\
\hline
\end{tabular}

Student $t$ test $p>0.05$

Table 2. Percentage (\%) and absolute number ( $n$ ) of sites positive for the periodontal pathogens Aggregatibacter actinomycetemcomitans $\mathrm{Aa}$, Porphyromonas gingivalis Pg and Tannerella forsythia Tf in ligatures from the control and RESV groups.

\begin{tabular}{lccc}
\hline Variable & $\mathrm{Aa}$ & $\mathrm{Pg}$ & Tf \\
\hline Control [\% (n)] & $83.3(10)$ & $50.0(6)$ & $41.7(5)$ \\
RESV [\% (n)] & $91.7(11)$ & $33.3(4)$ & $33.3(4)$ \\
p-value & 0.54 & 0.41 & 0.67 \\
\hline
\end{tabular}

Chi-squared test $p>0.05$. 


\section{Discussion}

Some evidence suggests that resveratrol may have antibacterial potential, including against periodontitis-associated bacteria species. ${ }^{1718}$ However, until now, no in vivo study has examined the influence of this substance on periodontal pathogens in sites presenting with periodontal breakdown. The present study, which used quantitative analyses and real-time PCR, represented an effort to determine the actual impact of resveratrol on the bacterial load of periodontal-related species during the progression of experimental periodontitis in rats.

In the current investigation, the animals did not show any signs of systemic illness throughout the study period. The rats also did not lose weight throughout the experimental period, and no deaths were observed. Inter-group comparisons of the morphometric results revealed higher bone-loss values in ligated molars and unligated teeth in the control group than the RESV group $(p<0.05)^{15}$. Concerning the microbiological outcomes observed in this study, inter-group comparisons revealed that no difference between groups was detected for Aa, Pg or Tf levels $(p>0.05)$. Similar results were observed regarding the percentage of sites positive for these bacteria after therapy, and there were no differences between groups ( $p>0.05)$. Thus, the findings of this investigation revealed that this natural plant compound did not promote additional benefits in terms of pathogen reduction in sites presenting with induced periodontal disease.

Previous studies have reported that resveratrol provides important antimicrobial actions, including antiviral, antifungal, antiprotozoal, and antibacterial activities. ${ }^{26,27,28,29}$ When the antimicrobial effect of resveratrol on the growth of periodontal pathogens was analyzed, $\mathrm{O}^{\prime} \mathrm{C}$ nnor et al. ${ }^{18}$ showed in vitro that this agent inhibited the anaerobic bacteria $\mathrm{Aa}$ and Pg in a similar time-dependent manner. Although the microbiological data of the current study have not demonstrated that this medicinal plant significantly interfered with the decrease of putative periodontopathogens, a trend toward the reduction of $A$. actinomycetemcomitans was observed in rats treated with resveratrol $(p=0.08)$ (Table 1). To the best of our knowledge, this is the first experimental study to investigate the antibacterial impact of resveratrol in pathogens related to periodontitis, which prevents a more direct comparison to the outcomes from other investigations.

Importantly, previous studies demonstrated that susceptibility to resveratrol may be different among bacteria, and this agent may present different antibacterial mechanisms of action according to the microorganism by exerting either a bacteriostatic or bactericidal effect. ${ }^{19,30,31,32,33}$ Indeed, although the study by O'Connor et al. ${ }^{18}$ had reported promising effects of resveratrol against anaerobic bacteria, its outcomes did not demonstrate encouraging results when aerobic microorganisms were evaluated. According to the authors, it is possible that the antioxidant properties of resveratrol inhibit the metabolic pathways of the bacteria that are mandatory for anaerobic survival. ${ }^{18}$ However, additional investigations are required to support this hypothesis.

It is important to highlight that the current study evaluated the antimicrobial ability of resveratrol on the periodontitis-associated species during biofilm formation and not in in vitro experiments, which may explain differences in the observed results when they are compared with the findings of $\mathrm{O}^{\prime} \mathrm{Connor}$ et al., ${ }^{18}$ which could be considered more similar to clinical conditions. Interestingly, the periodontopathic bacteria studied in the present study were responsible for the initiation of periodontal disease and may perpetuate the imbalance in the microbiota and the host inflammatory response in periodontal tissues to stimulate the progression of periodontitis. ${ }^{4}$ Similar to human periodontitis, bacterial penetration of the host tissue is thought to be a crucial factor affecting alveolar bone loss in rats. Rovin et al. ${ }^{34}$ assessed the influence of bacteria and their antigens on the beginning of periodontal disease in germ-free and conventional rats, and they showed that microorganisms were necessary for the occurrence of inflammatory periodontal disease. Nevertheless, it is not possible to guarantee that the species studied in this study act as late colonizers in rats because our results are limited to only one time point. However, it is essential to mention that the bacteria that were studied were probably not in the sites before the ligatures were placed. According to Duarte et al., ${ }^{6}$ none of the 40 species investigated in their research using checkerboard DNA-DNA hybridization, including those investigated in the present study, were detected in the sulcus of unligated teeth. Although 
rats are not natural hosts for many human bacteria, an exception to this general principle is infection with $A$. actinomycetemcomitans, which may naturally colonize the oral cavity of rats ${ }^{35}$. On the other hand, although $P$. gingivalis colonization has been described as not being abundantly present in the ligature model in rats, ${ }^{6}$ previous researchers reported that $A$. actinomycetemcomitans and P. gingivalis were found in ligature samples after 7 days of experimental periodontitis induction in rats, which agrees with our data ${ }^{36}$.

Concerning the experimental periodontitis model used in the present study, it is important to highlight that numerous experiments demonstrated the loss of periodontal attachment and the resorption of alveolar bone occurring predictably in a 7-day period in rats ${ }^{37}$, even though some investigations have been conducted for longer periods of time $e^{38}$. In the current investigation, the rationale for using eleven days as the experimental period was based on previous research that revealed that the application of this model of periodontitis induction can be recommended for only short periods (less than 15 days) ${ }^{23}$. Previous publications from our research group used this period of ligature (11 days), which agreed with the model used in the current investigation and demonstrated that the evaluated substances were able to promote advantages in alveolar bone loss and host immune-inflammatory response ${ }^{15}$. Importantly, the therapies were administered during 30 days, for 19 days before periodontitis induction and for another 11 days following ligature placement. Regarding microbial analysis, it should be highlighted that our study used a quantitative PCR technique that allowed for the detection of $10^{3}$ cells. In a probe hybridization technique, as performed in the study by Duarte et al. ${ }^{6}$ study, the lower limit is usually $10^{5}$, with samples considered to be negative in the presence of some pathogens if this threshold is not achieved. Thus, from the perspective of the more sensitive technique, the detection of these fastidious species could be favored in the present investigation, which would allow for an adequate analysis of periodontal pathogens during the progression of experimental periodontitis.

A limitation of the current study could be attributed to the type of administration employed to evaluate the antimicrobial performance of resveratrol in diseased periodontal tissues. Future research is needed to determine the microbiological impact of the local application of resveratrol, instead of its systemic use, on periodontal pathogens because previous studies demonstrated significant antimicrobial outcomes with the topical application of resveratrol in other infectious conditions. ${ }^{26,27}$ In addition, although different dosages and exposure times for resveratrol may provide additional effects for this substance on periodontal pathogens during experimental periodontitis in rats, the protocol employed in the current study was based on previous experiments using different animal models that demonstrated advantages for using resveratrol following this protocol. . $^{8,13,14,24}$

It is important to mention that no sample size calculation was performed in the present study, and the sample size was based on previous animal studies that also evaluated the effects of different substances on the progression of experimental periodontitis in rats. ${ }^{15,23,38}$. It may be hypothesized that a higher sample size could provide different outcomes and lead to significance for microbiological results during experimental periodontitis in rats.

Within the limitations of this investigation, it was demonstrated that the continuous use of resveratrol did not promote additional benefits in the microbiological outcomes of experimental periodontitis in rats, although a trend toward to the reduction of $A$. actinomycetemcomitans was observed in the rats that received resveratrol. Considering the previous data from the literature and the findings of the present study, the promising effects of resveratrol to reduce periodontal breakdown seem to be mediated mainly by its modulatory role in the immune-inflammatory response more than by its inhibitory effect on the periodontal pathogens. Taking into account the paradigm of periodontitis, periodontal pathogens are necessary for disease, although the magnitude and severity of periodontal damage are dependent on the individual hostimmune-inflammatory response to the bacterial challenge. Most of the therapeutic substances proposed as adjunctive to periodontal therapy have antibiotic properties or anti-inflammatory action. The employment of therapeutic strategies linking both the antibacterial and host-modulatory effects would improve the possibility of effectively treating periodontitis. 


\section{Conclusion}

This study demonstrated that resveratrol does not promote benefits for microbiological outcomes of an experimental model of periodontitis, which suggests there are other mechanisms through which this substance exerts its promising effects to control periodontitis.

\section{References}

1. Burt B. AAP Position paper: epidemiology of periodontal diseases. J Periodontol. 2005;76(8):1406-19. doi:10.1902/jop.2005.76.8.1406

2. Kinane DF, Preshaw PM, Loos BG. Host-response: understanding the cellular and molecular mechanisms of host-microbial interactions: consensus of the Seventh European Workshop on Periodontology. J Clin Periodontol. 2011;38 Suppl 11:44-8. doi:10.1111/j.1600-051X.2010.01682.x

3. Kornman KS. Mapping the pathogenesis of periodontitis: a new look. J Periodontol. 2008;79(8 Suppl):1560-8. doi:10.1902/jop.2008.080213

4. Socransky SS, Haffajee AD, Cugini MA, Smith C, Kent RL Jr. Microbial complexes in subgingival plaque. J Clin Periodontol. 1998;25(2):134-44. doi:10.1111/j.1600-051X.1998.tb02419.x

5. Ready D, D'Aiuto F, Spratt DA, Suvan J, Tonetti MS, Wilson M. Disease severity associated with presence in subgingival plaque of Porphyromonas gingivalis, Aggregatibacter actinomycetemcomitans, and Tannerella forsythia, singly or in combination, as detected by nested multiplex PCR. J Clin Microbiol. 2008;46(10):3380-3. doi:10.1128/JCM.01007-08

6. Duarte PM, Tezolin KR, Figueiredo LC, Feres M, Bastos MF. Microbial profile of ligature-induced periodontitis in rats. Arch Oral Biol. 2010;55(2):142-7. doi:10.1016/j.archoralbio.2009.10.006

7. Singh SU, Casper RF, Fritz PC, Sukhu B, Ganss B, Girard $\mathrm{B}$ Jr et al. Inhibition of dioxin effects on bone formation in vitro by a newly described aryl hydrocarbon receptor antagonist, resveratrol. J Endocrinol. 2000;167(1):183-95. doi:10.1677/joe.0.1670183

8. Schmatz R, Mazzanti CM, Spanevello R, Stefanello N, Gutierres J, Corrêa M, et al. Resveratrol prevents memory deficits and the increase in acetylcholinesterase activity in streptozotocin-induced diabetic rats. Eur J Pharmacol. 2009;610(1-3):42-8. doi:10.1016/j.ejphar.2009.03.032

9. Bhatt SR, Lokhandwala MF, Banday AA. Resveratrol prevents endothelial nitric oxide synthase uncoupling and attenuates development of hypertension in spontaneously hypertensive rats. Eur J Pharmacol. 2011;667(1-3):258-64. doi:10.1016/j.ejphar.2011.05.026

\section{Acknowledgments}

This study was supported by the São Paulo Research Foundation FAPESP, São Paulo, SP, Brazil - Processes 2012/50487-0 and the National Counsel of Technological and Scientific Development CNPq - Processes 303693/2009-6 and 118063/2012-0.

10. Soufi FG, Sheervalilou R, Vardiani M, Khalili M, Alipour MR. Chronic resveratrol administration has beneficial effects in experimental model of type 2 diabetic rats. Endocr Regul. 2012;46(2):83-90. doi:10.4149/endo_2012_02_83

11. Xuzhu G, Komai-Koma M, Leung BP, Howe HS, McSharry C, McInnes IB et al. Resveratrol modulates murine collagen-induced arthritis by inhibiting Th17 and B-cell function. Ann Rheum Dis. 2012;71(1):129-35 doi:10.1136/ard.2011.149831

12. Whitlock NC, Baek SJ. The anticancer effects of resveratrol: modulation of transcription factors. Nutr Cancer. 2012;64(4):493-502. doi:10.1080/01635581.2012.667862

13. Sharma S, Kulkarni SK, Chopra K. Effect of resveratrol, a polyphenolic phytoalexin, on thermal hyperalgesia in a mouse model of diabetic neuropathic pain. Fundam Clin Pharmacol. 2007;21(1):89-94. doi:10.1111/j.1472-8206.2006.00455.x

14. Gao X, Xu YX, Janakiraman N, Chapman RA, Gautam, SC. Immunomodulatory activity of resveratrol: suppression of lymphocyte proliferation, development of cell-mediated cytotoxicity, and cytokine production. Biochem Pharmacol. 2001;62(9):1299-308. doi:10.1016/S0006-2952(01)00775-4

15. Casati MZ, Algayer C, Cruz GC, Ribeiro FV, Casarin RC, Pimentel SP et al. Resveratrol decreases periodontal breakdown and modulates local levels of cytokines during periodontitis in rats. J Periodontol. 2013;84(10):e58-64. doi:10.1902/jop.2013.120746

16. Moridi H, Karimi J, Sheikh N, Goodarzi MT, Saidijam M, Yadegarazari R, et al. Resveratrol-dependent down-regulation of receptor for advanced glycation end-products and oxidative stress in kidney of rats with diabetes. Int J Endocrinol Metab. 2015;13(2):e23542. doi:10.5812/ijem.23542

17. Park EJ, Pezzuto JM. The pharmacology of resveratrol in animals and humans. Biochim Biophys Acta. 2015;1852(6):1071-113. doi:10.1016/j.bbadis.2015.01.014

18. O'Connor DJ, Wong RW, Rabie AB. Resveratrol inhibits periodontal pathogens in vitro. Phytother Res. 2011;25(11):1727-31. doi:10.1002/ptr.3501 
19. Ferreira S, Silva F, Queiroz JA, Oleastro M, Domingues FC. Resveratrol against Arcobacter butzleri and Arcobacter cryaerophilus: activity and effect on cellular functions. Int J Food Microbiol. 2014;180:62-8. doi:10.1016/j.ijfoodmicro.2014.04.004

20. Taylor EJ, Yu Y, Champer J, Kim J. Resveratrol demonstrates antimicrobial effects against propionibacterium acnes in vitro. Dermatol Ther (Heidelb). 2014;4(2):249-57. doi:10.1007/s13555-014-0063-0

21. Hwang D, Lim YH. Resveratrol antibacterial activity against Escherichia coli is mediated by Z-ring formation inhibition via suppression of FtsZ expression. Sci Rep. 2015;5:10029. doi:10.1038/srep10029

22. Rizzo A, Bevilacqua N, Guida L, Annunziata M, Romano Carratelli C, Paolillo R. Effect of resveratrol and modulation of cytokine production on human periodontal ligament cells. Cytokine. 2012;60(1):197-204. doi:10.1016/j.cyto.2012.06.004

23. Toker H, Ozan F, Ozer H, Ozdemir H, Eren K, Yeler H. A morphometric and histopathologic evaluation of the effects of propolis on alveolar bone loss in experimental periodontitis in rats. J Periodontol. 2008;79(6):1089-94. doi:10.1902/jop.2008.070462

24. Casarin RC, Casati MZ, Pimentel SP, Cirano FR, Algayer M, Pires PR et al. Resveratrol improves bone repair by modulation of bone morphogenetic proteins and osteopontin gene expression in rats. Int J Oral Maxillofac Surg. 2014l;43(7):900-6. doi:10.1016/j.ijom.2014.01.009

25. Clais S, Boulet G, Van Kerckhoven M, Lanckacker E, Delputte P, Maes L, et al. Comparison of viable plate count, turbidity measurement and real-time PCR for quantification of Porphyromonas gingivalis. Lett Appl Microbiol. 2015;60(1):79-84. doi:10.1111/lam.12341

26. Chan MM. Antimicrobial effect of resveratrol on dermatophytes and bacterial pathogens of the skin. Biochem Pharmacol. 2002;63(2):99-104. doi:/10.1016/S0006-2952(01)00886-3

27. Docherty JJ, Smith JS, Fu MM, Stoner T, Booth T. Effect of topically applied resveratrol on cutaneous herpes simplex virus infections in hairless mice. Antiviral Res. 2004;61(1):19-26. doi:10.1016/j.antiviral.2003.07.001

28. Kedzierski L, Curtis JM, Kaminska M, Jodynis-Liebert J, Murias M. In vitro antileishmanial activity of resveratrol and its hydroxylated analogues against Leishmania major promastigotes and amastigotes. Parasitol Res. 2007;102(1):91-7. doi:10.1007/s00436-007-0729-y
29. Wang WB, Lai HC, Hsueh PR, Chiou RY, Lin SB, Liaw SJ. Inhibition of swarming and virulence factor expression in Proteus mirabilis by resveratrol. J Med Microbiol. 2006;55(10):1313-21. doi:10.1099/jmm.0.46661-0

30. Paulo L, Oleastro M, Gallardo E, Queiroz JA, Domingues F. Anti-Helicobacter pylori and urease inhibitory activities of resveratrol and red wine. Food Res Int. 2011; 44(4):964-9. doi:10.1016/j.foodres.2011.02.017

31. Selma MV, Larrosa M, Beltrán D, Lucas R, Morales JC, Tomás-Barberán F et al. Resveratrol and some glucosyl, glucosylacyl, and glucuronide derivatives reduce Escherichia coli O157:H7, Salmonella Typhimurium, and Listeria monocytogenes Scott A adhesion to colonic epithelial cell lines. J Agric Food Chem. 2012;60(30):7367-74. doi:10.1021/jf203967u

32. Brown JC, Jiang, X. Activities of muscadine grape skin and poly phenolic constituents against Helicobacter pylori. J Appl Microbiol. 2013;114(4):982-91.

33. Nawrocki EM, Bedell HW, Humphreys TL. Resveratrol is cidal to both classes of Haemophilus ducreyi. Int J Antimicrob Agents. 2013;41(5):477-9. doi:10.1016/j.ijantimicag.2013.02.008

34. Rovin S, Costich ER, Gordon HA. The influence of bacteria and irritation in the initiation of periodontal disease in germfree and conventional rats. J Periodontal Res. 1966;1(3):193-204.

35. Fine DH, Velliyagounder K, Furgang D, Kaplan JB. The Actinobacillus actinomycetemcomitans auto-transporter adhesin Aae exhibits specificity for buccal epithelial cells from humans and old world primates. Infect Immun. 2005;73(4):1947-53.

36. Theodoro LH, Pires JR, Fernandes LA, Gualberto Júnior EC, Longo M, de Almeida JM, Garcia VG. Effect of antimicrobial photodynamic therapy on periodontally infected tooth sockets in rats. Lasers Med Sci. 2015;30(2):677-83.

37. Xie R, Kuijpers-Jagtman AM, Maltha JC. Inflammatory responses in two commonly used rat models for experimental tooth movement: comparison ith ligature-induced periodontitis. Arch Oral Biol. 2011; 56(2):159-67.

38. Bezerra JP1, Silva LR, Lemos VA, Duarte PM, Bastos MF. Administration of high doses of caffeine increases alveolar bone loss in ligature-induced periodontitis in rats. J Periodontol. 2008;79(12):2356-60. 